\title{
Visualización y Razonamiento Configural
}

\author{
Visualization and Configural Reasoning
}

\author{
Francisco Clemente \\ Salvador Llinares ${ }^{* *}$ \\ Germán Torregrosa ${ }^{* * * *}$
}

\begin{abstract}
Resumen
Esta investigación estudia la relación entre la identificación de figuras prototípicas y el conocimiento de geometría durante la resolución de problemas de probar como una manifestación del razonamiento configural. Se han analizado las respuestas de 182 estudiantes para maestro a dos problemas de probar que proporcionaban una configuración geométrica y pedían probar un hecho geométrico. Los resultados indican que la identificación de una figura prototípica en la configuración inicial tiene un efecto heurístico que activa determinados conocimientos de geometría, que favorecen el cambio del anclaje visual al anclaje discursivo en la resolución del problema. Estos resultados subrayan la importancia de la relación entre la visualización y el conocimiento geométrico en el desarrollo del razonamiento configural durante la resolución de problemas de probar. Finalmente, desde estos resultados generamos algunas implicaciones para la formación de los maestros.
\end{abstract}

Palabras clave: Razonamiento Configural. Prueba. Figura prototípica. Aprendizaje de la geometría.

\begin{abstract}
This research analyses the relationships between the identification of prototypical figures and geometrical knowledge in solving proof problems as a evidence of configural reasoning. We analyze the answers of 182 preservice teachers on two geometrical proof problems, which include a geometrical configuration and a request for proving a geometrical fact. The results indicate that identifying a prototypical figure in the initial configuration has a heuristic effect that determines the path of problem-solving, activating certain geometrical facts and generating the change from visual anchor to anchor discursive. These results allow us to character the relationship between visualization and geometric knowledge in the configural reasoning during proof problem solving. Finally, we derive some implications for teacher education.
\end{abstract}

Keywords: Configural Reasoning. Proof. Prototypical Figure. Visualization. Geometry Learning.

\section{Introducción}

\footnotetext{
" Doctor por la Universidad de Alicante (UA). Profesor Colaborador honorifico en el Departamento de Innovación y Formación Didáctica, Facultad de Educación de la Universidad de Alicante (UA), Alicante, España. Dirección Postal: Campus de Sant Vicent del Raspeig, 03080 Alicante, España. E-mail: fclemcis@gmail.com.

*** Doctor en Filosofía y Ciencias de la Educación de la Universidad de Sevilla (US). Catedrático de Universidad en el Departamento de Innovación y Formación Didáctica de la Facultad de Educación, Universidad de Alicante (UA). Dirección Postal: Campus de Sant Vicent del Raspeig, 03080, Alicante, España. E-mail: sllinares@ua.es.

*** Doctor en Matemáticas por la Universidad de Alicante (UA). Catedrático de Escuela Universitaria de la Universidad de Alicante (UA), Departamento de Innovación y Formación Didáctica, Facultad de Educación. Dirección Postal: Campus de Sant Vicent del Raspeig, 03080, Alicante, España. E-mail: german.torregrosa@ua.es.
} 
El análisis de la relación entre los procesos de visualización y el conocimiento de geometría en la resolución de problemas de probar ha puesto de manifiesto las dificultades que tienen algunos resolutores para usar el conocimiento de geometría previamente aprendido (BATTISTA, 2007; CHINNAPPAN; LAWSON, 2005; GAL; LINCHEVSKI, 2010). En estas situaciones, Duval $(1995,2007)$ y Fischbein (1993) han subrayado el papel heurístico que desempeñan las figuras en la resolución de los problemas en los que se proporciona una configuración geométrica. En la resolución de estos problemas, los procesos de exploración e indagación ponen de relieve los vínculos entre el conocimiento de geometría y los procesos de visualización (HANNA; SIDOLI, 2007; HERSHKOWITZ, 1990; PRESMEG, 2006).

Fischbein (1993) mantiene que las figuras geométricas poseen, al mismo tiempo, aspectos figurales y conceptuales. En los problemas de probar es posible usar simultáneamente estos aspectos, ya que las propiedades de las figuras geométricas (representaciones) proceden de las definiciones y relaciones geométricas usadas en su construcción. Duval $(1998,1995)$ denomina aprehensión operativa a la modificación de una figura para considerar subconfiguraciones, añadiendo o quitando nuevos elementos geométricos, manipulando las diferentes partes de una configuración geométrica como un puzle para fijar la atención sobre subconfiguraciones particulares, o simplemente, fijando la atención sobre una parte específica de la configuración.

Por otra parte, con la aprehensión discursiva se asocian afirmaciones matemáticas a una configuración. Durante el proceso de resolución de un problema, los hechos geométricos identificados en la configuración se pueden relacionar con otros hechos geométricos y ser considerados premisas de alguna relación geométrica previamente conocida; es decir, generan una conjetura basada en lo visual que sugieren ideas que pueden ser usadas en la generación de relaciones deductivas. Este proceso que relaciona la visualización y el conocimiento de geometría ha sido denominado razonamiento configural (PRIOR; TORREGROSA, 2013; TORREGROSA; QUESADA, 2007; TORREGROSA; QUESADA; PENALVA, 2010).

El análisis del razonamiento configural muestra la importancia de la visualización en el desarrollo de los procesos deductivos en la resolución de los problemas de probar en geometría en los que se proporciona una configuración geométrica. La resolución de estos problemas implica un proceso de ver apoyado en la percepción (GAL; LINCHEVSKI, 2010). Estas investigaciones previas han puesto de manifiesto dos características de la relación entre la visualización y el conocimiento de geometría del resolutor: (i) el reconocimiento de una subconfiguración relevante en una configuración geométrica es una condición necesaria pero no suficiente para desencadenar el razonamiento configural, y (ii) la generación de un proceso 
deductivo se apoya en el conocimiento previo de algunos hechos geométricos y a las aprehensiones discursivas vinculadas a la configuración dada (CLEMENTE; LLINARES, 2013, 2014, 2015; LLINARES; CLEMENTE, 2014; CLEMENTE; TORREGROSA; LLINARES, 2015).

Estas características han permitido reconocer el papel relevante de la visualización en la generación de los procesos deductivos y, también, que un hecho geométrico puede desempeñar papeles diferentes en el proceso de resolución de este tipo de problemas (HERBST, 2004; HERBST et al., 2009). Este hecho permite diferenciar las interacciones de los estudiantes con las configuraciones que permiten construir conjeturas razonadas y usar razonamiento deductivo para averiguar lo que podría o debería ser verdad (HERBST, 2004).

Es decir, se subraya la importancia de los procesos previos al desarrollo de un proceso puramente deductivo en la resolución de los problemas. Estos procesos previos son los que se construyen sobre la relación entre la visualización y las aprehensiones operativas y discursivas que constituyen el razonamiento configural. En este trabajo, nosotros pretendemos analizar y explicar la relación que existe entre la identificación de figuras prototípicas y el conocimiento de geometría que es previa a la generación de un proceso puramente deductivo.

La imagen prototípica de las figuras geométricas que los estudiantes han generado a lo largo de su experiencia escolar influye en su capacidad de reconocerlas o de construir determinados objetos geométricos durante la resolución de los problemas (MESQUITA, 1998; VINNER; HERSHKOWITZ, 1980). La importancia de la tipicalidad de las representaciones geométricas viene dada porque influye en el papel de las representaciones externas en el aprendizaje de la geometría. En el caso particular de las configuraciones en los problemas de probar en geometría, la importancia de la identificación de una configuración prototípica, durante su resolución, radica en que ayuda a activar algunos conocimientos de geometría frente a otros. En este sentido, en la medida en la que el resolutor identifique una configuración prototípica que esté vinculada a ítems de conocimientos relacionados entre sí estará en mejor condición de iniciar el razonamiento configural (GAL; LINCHEVSKI, 2010)

Para Hollebrands, Laborde y Stráber (2008) las configuraciones en geometría, tanto en entornos de lápiz y papel como en software de geometría dinámica (DGS: Dynamical Geometry Software), permiten un camino adicional en el aprendizaje ya que pueden presentar multitud de relaciones geométricas. En este sentido, la fortaleza del resolutor experto radica en sus habilidades para pasar de la configuración al discurso y reconocer formas y configuraciones vinculadas al razonamiento deductivo basado en conocimientos teóricos. Sin embargo, esta práctica no es espontánea para los estudiantes inexpertos. 
En relación a este último punto, Chen y Herbst (2013) indican que la naturaleza de las configuraciones (por ejemplo, ausencia de etiqueta para indicar el punto de intersección de dos segmentos) podría desempeñar un papel importante en el razonamiento geométrico de los estudiantes al condicionar la posibilidad de hacer conjeturas razonadas. La configuración inicialmente presentada en un problema geométrico puede contribuir a la manera en la que los resolutores lo estructuran para iniciar su resolución al apoyar la intuición geométrica, ya que les puede ayudar a relacionar los objetos geométricos.

Sin embargo, algunas veces este apoyo visual hace evidente a los resolutores relaciones no verdaderas, lo que puede llegar a abortar el desarrollo de un razonamiento configural adecuado. La idea de la tipicalidad de una representación (HERSHKOWITZ, 1990) intenta poner de relieve la influencia que tiene el uso de figuras prototípicas para mostrar más claramente algún atributo geométrico. Por ejemplo, las dificultades que tienen algunos estudiantes en la identificación de triángulos rectángulos cuando sus lados perpendiculares dejaban de ser paralelos a los márgenes del papel (HERSHKOWITZ, 1989).

En este contexto es en el que Mesquita (1998) denominó figura prototípica a aquella que tiende a respetar algunas propiedades, como privilegiando algunas líneas frente a otras (por ejemplo, en la representación del triángulo teniendo un lado paralelo al borde de la hoja), formas que tienden a ser regulares, simples y simétricas (MESQUITA, 1998). Para Gal y Linchevski (2010) el proceso de reconocimiento visual de figuras prototípicas puede ser explicado como el resultado del análisis de diversas características, en el que el objeto se segmenta en un conjunto de subobjetos (por ejemplo, la identificación de cuatro triángulos formados por las diagonales de un cuadrilátero). Cuando se reconocen las piezas que forman el objeto y su configuración, el objeto se reconoce como un patrón compuesto de estas piezas.

Por todo lo anterior, en el contexto particular de los problemas de probar en geometría, para los estudiantes no es fácil el paso de la argumentación basada en consideraciones intuitivas y visuales a consideraciones lógico-deductivas (PRUSAK; HERSHKOWITZ; SCHWARZ, 2012), por ello, es importante caracterizar las condiciones que favorezcan la transición entre lo visual y la generación de un proceso deductivo.

En los problemas de probar en los que se proporciona una configuración inicial, la importancia de la identificación de una subconfiguración relevante para la resolución del problema reside en que refuerza la activación de ciertos conocimientos de geometría. Consecuentemente, determinar cómo la identificación de una configuración permite movilizar determinados ítems de conocimiento, puede aportar información sobre la naturaleza de la 
relación entre la visualización y el conocimiento usado de manera productiva durante la resolución del problema (CHINNAPPAN, 1998) al iniciar el razonamiento configural.

Considerando estas referencias previas, esta investigación se ha centrado en caracterizar el desarrollo del razonamiento configural, en particular analizando las relaciones entre (i) la identificación de figuras prototípicas y (ii) el conocimiento de geometría que permite generar conjeturas razonadas.

\section{Marco teórico}

En esta investigación hemos adoptado el significado de concepto figural de Fischbein (1993) y la perspectiva cognitiva de Duval (1995, 1998, 1999) sobre el aprendizaje de la geometría. El significado que atribuimos a la visualización se refiere a lo indicado por Hershkowitz, Parzysz y Van Dormolen (1996, p.163): “entendemos por visualización la transferencia de objetos, conceptos, fenómenos, procesos y sus representaciones a algún tipo de representación visual y viceversa. Esto incluye también la transferencia de un tipo de representación visual a otra”. Siguiendo a Mesquita (1998), denominamos figura a la representación externa de una situación geométrica.

Fischbein (1993) define una nueva entidad mental que denomina concepto figural, que hace depender la consideración de relaciones en las figuras a las definiciones y teoremas previamente conocidos. Fischbein indica que inferir información adicional sobre la configuración no procede de considerar de manera separada la figura y las relaciones lógicas entre los hechos geométricos, sino de un único proceso en el que la figura se ve de otra manera permitiendo revelar relaciones lógicas. Fischbein caracteriza este proceso, indicando que la figura no es una imagen ordinaria, sino una estructura controlada lógicamente.

Para Duval (1995) las asociaciones de afirmaciones matemáticas a configuraciones o subconfiguraciones, que constituyen las aprehensiones discursivas que caracterizan este control lógico de las figuras, se pueden realizar de dos maneras según el sentido de la transferencia realizada: del anclaje visual al anclaje discursivo (a un dibujo se le asocian afirmaciones matemáticas) y del anclaje discursivo al anclaje visual (ante una afirmación matemática el estudiante tiene la capacidad para realizar un dibujo que cumpla alguna de las características de la afirmación enunciada).

En este ámbito, la idea de Razonamiento Configural es entendida como la coordinación de las aprehensiones operativas y discursivas, que nos ayuda a comprender mejor cómo los estudiantes resuelven problemas de probar y el rol que desempeña la relación 
entre lo visual y el conocimiento de geometría en el desarrollo de razonamientos deductivos (TORREGROSA; QUESADA, 2007; TORREGROSA; QUESADA; PENALVA, 2010).

La figura prototípica que el estudiante es capaz de identificar desempeña un papel relevante en la coordinación de estas aprehensiones y, por tanto, en el inicio del razonamiento configural que se desencadena mediante la identificación de una figura prototípica. Las figuras prototípicas son representaciones que corresponden a una organización regular del contorno, orientación y forma. Se perciben preferentemente contornos cerrados; direcciones horizontales y verticales; formas regulares, simples y simétricas; así como componentes de la figura (lados y ángulos) que tienen dimensiones similares (MESQUITA, 1998).

Todas estas características consiguen reforzar la percepción de unas determinadas configuraciones respecto a otras y pueden tener un efecto heurístico, ya que incrementan la visibilidad de una figura particular y sus posibilidades de reorganización, que puede desempeñar un papel esencial en la búsqueda de una solución en un problema de geometría. Esto quiere decir que, de entre las varias figuras que el estudiante puede observar en la configuración inicial, la que logra llamar su atención estará vinculada con alguna de las figuras prototípicas que guarda en su memoria. Por ejemplo (Figura 1), los estudiantes pueden tener dificultades en reconocer, en la Figura 1a, el triángulo $\mathrm{ABC}$ como un triángulo isósceles, ya que la figura prototípica de triángulo isósceles suele ser la que aparece en la Figura 1b.

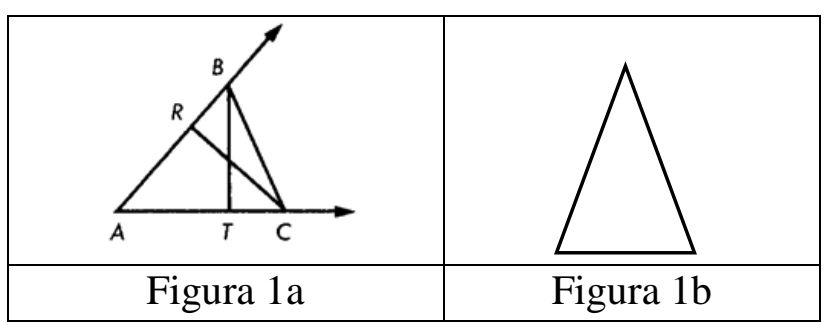

Figura 1- Diferentes representaciones de un triángulo isósceles. Fuente: adaptado de Moise y Downs (1986).

En las situaciones en las que hay que generar una prueba, y en las que hay que establecer algún vínculo entre la percepción visual y la argumentación lógico-deductiva, se plantea la necesidad de estudiar el papel que desempeña la identificación de una subconfiguración particular a partir de la cual se inician las coordinaciones entre las aprehensiones discursivas y operativas, que constituyen el razonamiento configural.

Considerando lo anterior, planteamos la siguiente cuestión de investigación centrada en caracterizar la relación entre la identificación de una subconfiguración, los conocimientos de geometría activados y la trayectoria de resolución seguida. De manera específica: ¿Qué relaciones existen entre la identificación de figuras prototípicas y el conocimiento de 
geometría activado que permite iniciar el razonamiento configural durante la resolución de problemas de probar?

\section{Método}

\subsection{Participantes}

En este estudio participaron 182 estudiantes para maestro que habían cursado una asignatura sobre Sentido Geométrico, organizada considerando los procesos cognitivos de visualización, construcción y prueba (DUVAL, 1995). Parte del contenido y las actividades en esta asignatura estaban dirigidas a aprender a visualizar propiedades geométricas de figuras planas como polígonos, triángulos, cuadriláteros y generar procesos de construir y probar. El objetivo del curso era que los estudiantes para maestro reconocieran propiedades geométricas mediante aprehensiones discursivas y operativas (DUVAL, 2007), utilizando diversos conceptos geométricos del currículo de educación primaria y desarrollaran el razonamiento configural.

\subsection{Instrumentos}

Como parte de la evaluación del curso se pidió a los estudiantes que resolvieran dos problemas de probar (Figura 2).

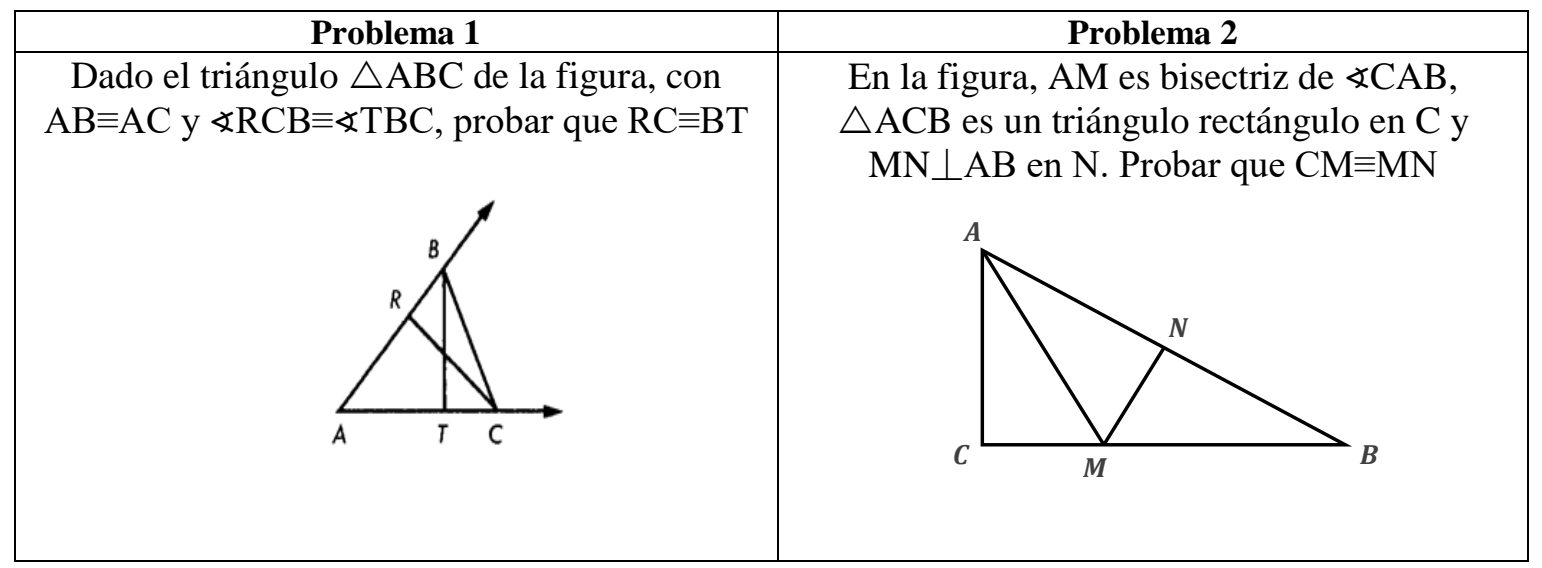

Figura 2 - Problemas de probar.

Fuente: Moise y Downs (1986).

En los problemas se presentaba una configuración geométrica e información de algunos hechos geométricos vinculados a la configuración. Los problemas pedían probar la congruencia de dos segmentos en la configuración dada. Para su resolución, los estudiantes 
debían desarrollar aprehensiones operativas y discursivas para identificar alguna subconfiguración que les permitiera reconocer hechos geométricos que podían relacionar para generar la prueba.

Previamente, se pidió a un grupo de formadores de maestros que presentaran diferentes alternativas para la resolución de los problemas en el contexto en el que se encuentran los resolutores, con el objetivo de identificar los hechos geométricos que definían los problemas y que podrían usarse para resolverlos. En el Cuadro 1 indicamos, primero, los elementos geométricos que podían proceder de realizar asociaciones directas de elementos geométricos a la configuración a partir de los datos del problema; y, en segundo lugar, los elementos geométricos susceptibles de ser usados para inferir información adicional.

\begin{tabular}{|c|c|}
\hline Problema 1 & Problema 2 \\
\hline $\begin{array}{l}\text { Asociación directa de elementos geométricos a la } \\
\text { configuración: } \\
\text { - (CA1) Definición de triángulo } \\
\text { - (CA4) Ángulos opuestos por el vértice son iguales } \\
\text { Elementos geométricos susceptibles de ser usados para } \\
\text { inferir información adicional: } \\
\text { - (CA2) Caracterización de triángulo isósceles (dos } \\
\text { lados congruentes y, por tanto, dos ángulos } \\
\text { congruentes). En un triángulo, los ángulos opuestos } \\
\text { a dos lados congruentes son congruentes y los lados } \\
\text { opuestos a dos ángulos congruentes son congruentes } \\
\text { - (CA3) Si a dos ángulos congruentes se les resta la } \\
\text { misma parte lo que queda son ángulos congruentes } \\
\text { - (CA9) Criterio de congruencia de triángulos A-L-A }\end{array}$ & $\begin{array}{l}\text { Asociación directa de elementos geométricos a la } \\
\text { configuración: } \\
\text { - (CA1) Definición de triángulo } \\
\text { - (CA5) Definición de bisectriz de un ángulo (es la } \\
\text { semirrecta que pasa por el vértice del ángulo y lo } \\
\text { divide en dos partes congruentes) } \\
\text { - (CA6) Definición de rectas perpendiculares } \\
\text { - (CA7) Definición de triángulo rectángulo } \\
\text { Elementos geométricos susceptibles de ser usados } \\
\text { para inferir información adicional: } \\
\text { - (CA8) La suma de los ángulos interiores de un } \\
\text { triángulo es } 180^{\circ} \text { (conocidos dos ángulos en un } \\
\text { triángulo, conocemos el tercero) } \\
\text { - (CA9) Criterio de congruencia de triángulos } \\
\text { A-L-A }\end{array}$ \\
\hline
\end{tabular}

Cuadro 1 - Conocimiento geométrico susceptible de ser utilizado en ambos problemas (CAi= código usado para indicar el ítem de conocimiento geométrico susceptible de ser usado en algún momento de la resolución de los problemas).

Fuente: autores.

En las configuraciones iniciales de los dos problemas se pueden identificar subconfiguraciones a partir de las cuales se pueden generar diferentes trayectorias de resolución (MESQUITA, 1998). En la Figura 3 mostramos las posibles subconfiguraciones relevantes que podían ser identificadas mediante alguna aprehensión operativa y que permitían reconocer triángulos con ángulos congruentes y lados congruentes, como premisas de alguno de los criterios de congruencia de triángulos. 


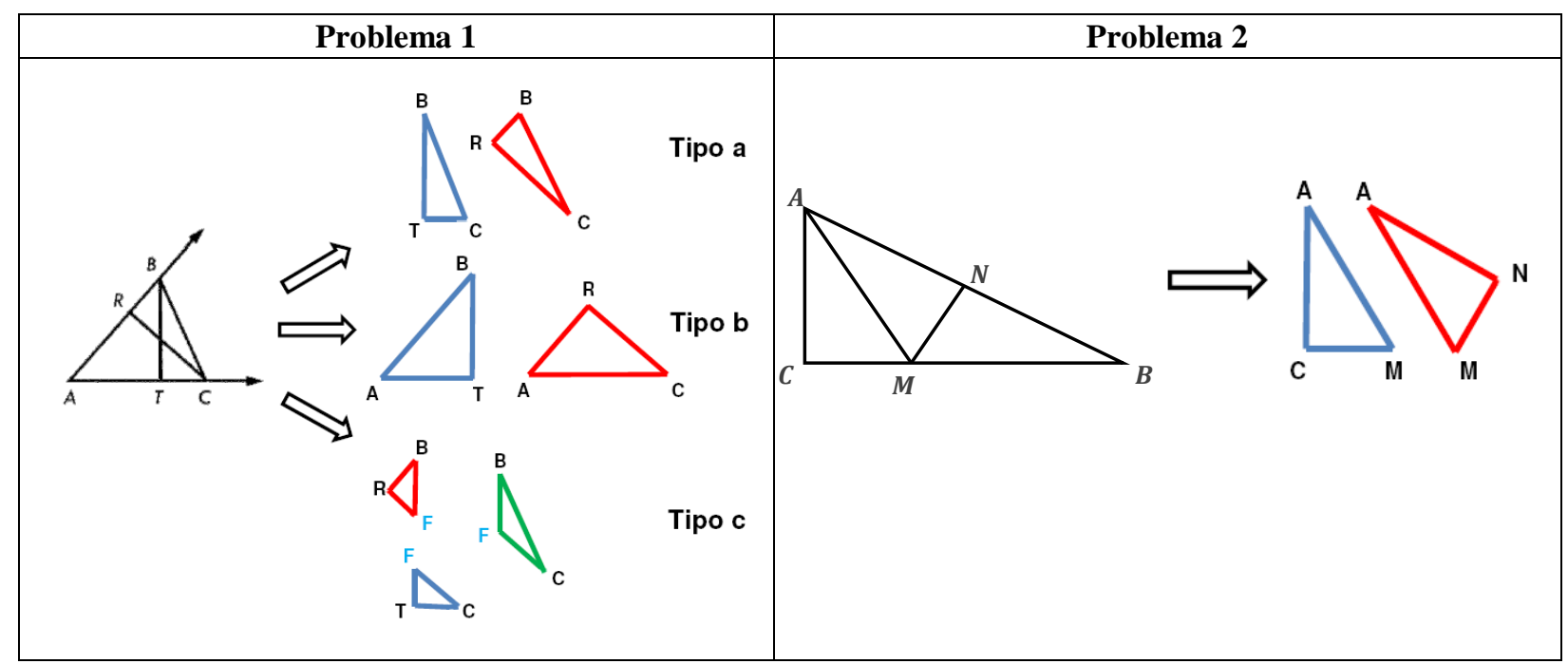

Figura 3 - Posibles subconfiguraciones consideradas en la resolución de los problemas.

Fuente: autores.

Las subconfiguraciones relevantes de los dos problemas (tres en el problema 1 y una en el problema 2) tienen características heurísticas diferentes. Las subconfiguraciones $a$ y $b$ en el problema 1 se solapan en la configuración inicial por lo que requieren una aprehensión operativa para visualizarlas por separado; mientras que la subconfiguración $c$ en el problema 1 y la única subconfiguración del problema 2 podían ser consideradas parte de la configuración inicial. Además, la subconfiguración $c$ en el problema 1 carece de etiqueta para indicar el punto de intersección F de los segmentos RC y BT lo que podría dificultar su identificación (CHEN; HERBST, 2013). Asimismo, en la única subconfiguración del problema 2, el triángulo rectángulo $\triangle \mathrm{ACM}$ se muestra en su representación prototípica (sus lados perpendiculares son paralelos a los bordes del papel en que está dibujado), lo que facilitaría su identificación (HERSHKOWITZ, 1989).

\subsection{Análisis}

Los datos usados en esta investigación son las respuestas dadas por los estudiantes a los dos problemas (un total de 364 respuestas). Inicialmente, en el discurso textual generado en cada resolución identificamos cómo el estudiante estaba construyendo su argumentación como consecuencia de algunas aprehensiones operativas y discursivas y su coordinación. El análisis se realizó en dos fases (LLINARES; CLEMENTE, 2014). En la fase 1, identificamos:

- Evidencias de que los estudiantes han generado alguna aprehensión operativa identificando alguna sub-configuración. Por ejemplo, en el problema 1 y según la 
subconfiguración reconocida, cuando el estudiante indica de alguna manera que está considerando los triángulos $\triangle \mathrm{RCB}$ y $\triangle \mathrm{TBC}$ (subconfiguración a); $\triangle \mathrm{ATB}$ y $\triangle \mathrm{ARC}$ (subconfiguración b); o bien, $\triangle \mathrm{CFB}, \triangle \mathrm{RFB}$ y $\triangle \mathrm{TFC}$ (subconfiguración c). Análogamente, en el problema 2 cuando tienen en cuenta los triángulos $\triangle \mathrm{ACM} y$ $\triangle \mathrm{AMN}$.

- Si los estudiantes reconocían los objetos geométricos dados como datos del problema en la configuración (aprehensión discursiva desde el anclaje discursivo al anclaje visual). Por ejemplo, si en el problema 1 y para la subconfiguración c, reconocían que los ángulos $\Varangle \mathrm{RFB}$ y $\Varangle \mathrm{TFC}$ son congruentes por ser opuestos por el vértice F. De la misma manera, en el problema 2 si indicaba que la bisectriz del ángulo $\Varangle \mathrm{CAB}$ determinaba dos ángulos iguales en $\Varangle \mathrm{A}$; que el ángulo $\Varangle \mathrm{C}$ era recto por ser $\triangle \mathrm{ACB}$ un triángulo rectángulo en $\mathrm{C}$; $\mathrm{y}$ que el ángulo $\Varangle \mathrm{N}$ es recto por ser $\mathrm{MN}$ perpendicular a $\mathrm{AB}$.

En la fase 2, identificamos:

- Si los estudiantes usaban un conocimiento externo a los datos del problema para generar información adicional. Por ejemplo, en el problema 1,

$\checkmark$ si utilizaban el hecho de que si el triángulo $\triangle \mathrm{ACB}$ tiene los lados $\mathrm{AB}$ y $\mathrm{AC}$ congruentes implica que es isósceles, y por tanto, los ángulos $\Varangle \mathrm{TCB}$ y $\Varangle \mathrm{RBC}$ también serán congruentes;

$\checkmark$ si el triángulo $\triangle \mathrm{CFB}$ tiene los ángulos $\Varangle \mathrm{FBC}$ y $\Varangle \mathrm{FCB}$ congruentes (dato del problema, ya que estos ángulos son los mismos que $\Varangle \mathrm{RCB}$ y $\Varangle \mathrm{TBC}$ ), implica que es isósceles y, por tanto, los lados BF y CF también serán congruentes;

$\checkmark$ si a dos ángulos congruentes ( $\Varangle \mathrm{TCB}$ y $\Varangle \mathrm{RBC}$ ) se les resta la misma parte ( $\Varangle \mathrm{RCB}$ y $\Varangle \mathrm{TBC})$, lo que queda son ángulos congruentes ( $\Varangle \mathrm{BAT}$ y $\Varangle \mathrm{CAR})$.

Análogamente, en el problema 2,

$\checkmark$ si utilizaban el hecho de que los ángulos interiores de un triángulo suman $180^{\circ} \mathrm{y}$, por tanto, conocidos dos ángulos en un triángulo podemos conocer el tercero.

- Si los estudiantes usaban un hecho geométrico externo a los datos del problema para generar información adicional sobre la configuración, utilizando información obtenida previamente como premisas en una cadena deductiva. Por ejemplo, si usaban el criterio de congruencia de triángulos A-L-A al reconocer la información reunida en la configuración como premisas de este criterio de congruencia.

De este modo, pudimos identificar los momentos en los que los ítems de conocimiento pueden desempeñar papeles diferentes, desde lo configural a desempeñar un estatus lógico en 
un proceso deductivo, y la manera en la que los estudiantes usaban las configuraciones en cada una de las trayectorias de resolución seguidas.

\section{Resultados}

Los dos problemas tuvieron niveles de éxito diferentes (Cuadro 2).

\begin{tabular}{|c|c|c|c|c|c|c|}
\hline \multirow{2}{*}{ Problema } & $\begin{array}{c}\text { Subconfi- } \\
\text { guración }\end{array}$ & $\begin{array}{c}\text { Conocimientos de geometría } \\
\text { requeridos }\end{array}$ & Estudiantes & Total & \multicolumn{2}{|c|}{$\%$ éxito } \\
\hline \multirow{3}{*}{ P1 } & $\mathrm{a}$ & CA1+CA2+CA9 & 116 & \multirow{3}{*}{164} & $39,7 \%$ & $66,7 \%$ \\
\cline { 2 - 4 } & $\mathrm{b}$ & CA1+CA2+CA3+CA9 & 39 & \\
\cline { 2 - 4 } & $\mathrm{c}$ & CA1+CA2+CA3+CA4+CA9 & 9 & & $33,3 \%$ & \\
\hline P2 & Única & CA1+CA5+CA6+CA7+CA8+CA9 & 165 & 165 & & $57 \%$ \\
\hline
\end{tabular}

Cuadro 2 - Relación entre la subconfiguración identificada y la trayectoria de resolución seguida $(\mathrm{CAi}=$ ítems de conocimiento geométrico activo en la trayectoria de resolución seguida según han sido dados en el Cuadro 1). Fuente: autores.

En el problema 1, de los 182 participantes, 164 estudiantes siguieron trayectorias de resolución vinculadas a alguna de las tres subconfiguraciones relevantes y 75 consiguieron generar procesos deductivos correctos $(45,7 \%, 75$ de 164). Los 18 participantes restantes no iniciaron la resolución del problema. En las tres trayectorias de resolución identificadas $(a, b y$ c) que generaron procesos deductivos correctos activaron respectivamente tres, cuatro y cinco ítems de conocimiento geométrico. Los porcentajes de éxito fueron 39,7\% (trayectoria vinculada a la subconfiguración $a$ ), 66,7\% (trayectoria vinculada a la subconfiguración $b$ ) y $33,3 \%$ (trayectoria vinculada a la subconfiguración c). En el problema 2, de los 182 participantes, 165 estudiantes siguieron la trayectoria de resolución definida por la subconfiguración relevante y 94 de estos generaron procesos deductivos correctos $(57 \%, 94$ de 165). Los otros 17 participantes no iniciaron la resolución del problema.

\subsection{Identificación de una subconfiguración y el conocimiento de geometría en la trayectoria de resolución}

La resolución seguida por los estudiantes fue caracterizada considerando la relación entre la identificación de una determinada subconfiguración y el uso del conocimiento de geometría puesto de manifiesto en la trayectoria de resolución seguida. Las características de los problemas usados permitían subrayar este aspecto ya que se diferenciaban en el número de subconfiguraciones que permitían generar trayectorias de resolución. En el problema 1, tres subconfiguraciones relevantes y en el problema 2 solo una. La trayectoria de resolución vinculada a la subconfiguración $a$ requiere usar (al menos) tres conocimientos de geometría, 
la vinculada a la subconfiguración $b$ requiere usar cuatro (los tres anteriores más la propiedad aditiva de los ángulos congruentes, CA3) y la vinculada a la subconfiguración $c$ requiere usar cinco (los cuatro anteriores más ángulos opuestos por el vértice son congruentes, CA4) (Cuadro 2).

El número de estudiantes que iniciaron cada una de las tres trayectorias de resolución en el problema 1 han sido 164 (116 para la subconfiguración $a$, 39 para la subconfiguración $b$ y 9 para la subconfiguración c). En el problema 2, 165 estudiantes identificaron la subconfiguración relevante siguiendo una trayectoria de resolución que implicaba la activación de seis ítems de conocimiento geométrico.

\subsection{Influencia de las figuras prototípicas en el inicio del razonamiento configural}

Nuestro análisis ha permitido identificar cuatro comportamientos relativos a la influencia de las figuras prototípicas en el inicio del razonamiento configural que ponen de manifiesto el papel que desempeña la percepción visual en la activación del conocimiento (Cuadro 3).

\begin{tabular}{|c|c|c|c|c|c|}
\hline Problema & Caso & $\begin{array}{c}\text { Figura } \\
\text { prototípica }\end{array}$ & Descripción & $\begin{array}{l}\text { Subconfi- } \\
\text { guración }\end{array}$ & Frecuencia \\
\hline \multirow{5}{*}{$\mathrm{P} 1$} & 1 & $\begin{array}{l}\text { Triángulo } \\
\text { isósceles }\end{array}$ & Rotación de la figura inicial & $\mathrm{b}$ & 9 \\
\hline & \multirow[b]{2}{*}{2} & \multirow{2}{*}{$\begin{array}{l}\text { Ángulos } \\
\text { opuestos por } \\
\text { el vértice }\end{array}$} & \multirow{2}{*}{$\begin{array}{c}\text { Identifica el punto de intersección de los } \\
\text { segmentos TB y CR y sus ángulos } \\
\text { correspondientes }\end{array}$} & $\mathrm{a}$ & $2^{(1)}$ \\
\hline & & & & $\mathrm{c}$ & 7 \\
\hline & \multirow[b]{2}{*}{3} & \multirow{2}{*}{$\begin{array}{l}\text { Triángulo } \\
\text { rectángulo }\end{array}$} & \multirow{2}{*}{$\begin{array}{l}\text { Admite explícitamente una propiedad } \\
\text { errónea (AC } \perp \mathrm{BT} \text { y } \mathrm{RC} \perp \mathrm{AB}) \text { y marca } \\
\text { los ángulos rectos. Prioriza el aspecto } \\
\text { intuitivo frente al formal por las } \\
\text { características de la figura inicial }\end{array}$} & a & 10 \\
\hline & & & & $\mathrm{b}$ & 3 \\
\hline $\mathrm{P} 2$ & 4 & $\begin{array}{l}\text { Triángulo } \\
\text { rectángulo }\end{array}$ & $\begin{array}{l}\text { Rotación de una de las configuraciones } \\
(\triangle \mathrm{AMN}) \text { representándola con los lados } \\
\text { perpendiculares paralelos al borde del } \\
\text { papel }\end{array}$ & Única & 18 \\
\hline
\end{tabular}

Cuadro 3 - Comportamientos derivados de la influencia de las figuras prototípicas identificadas

(1) Estos dos estudiantes posteriormente también identifican la subconfiguración $a$ continuando con esta trayectoria de resolución;

Fuente: autores.

La importancia de estos comportamientos está en la información que proporcionan para determinar la manera en que la visualización y el conocimiento intervienen en la generación del razonamiento configural. En relación al problema 1, identificamos tres comportamientos característicos vinculados a la influencia de las figuras prototípicas triángulo isósceles, ángulos opuestos por el vértice y triángulo rectángulo (casos 1, 2 y 3). En relación al problema 2, identificamos un comportamiento característico vinculado a la 
influencia de la figura prototípica triángulo rectángulo (caso 4). Las características de estos distintos casos se describen a continuación.

- Caso 1: Influencia de la figura prototípica triángulos isósceles en el problema 1. Figura prototípica de triángulo isósceles: rotación de la figura inicial.

De los 39 alumnos que en el problema 1 escogieron la subconfiguración $b, 9$ realizaron una rotación de la figura inicial mediante una aprehensión operativa para obtener la representación prototípica de triángulo isósceles (Figura 4). Esta manera de proceder no se ha observado en los estudiantes que han escogido las subconfiguraciones $a$ y $c$. La Figura 4 muestra las representaciones realizadas por un estudiante que refleja la rotación de la figura inicial permitiéndole identificar subconfiguración $b$.

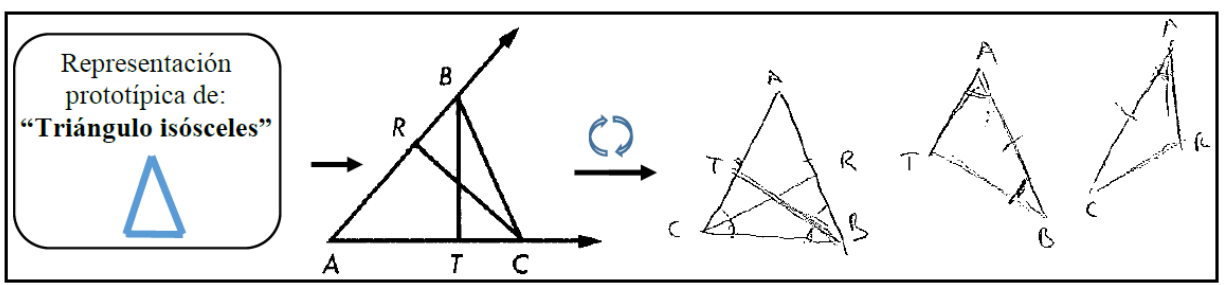

Figura 4 - Fragmento de la respuesta de un estudiante al problema 1 reflejando la influencia de la figura prototípica triángulo isósceles.

Fuente: autores.

- Caso 2: Influencia de la figura prototípica ángulos opuestos por el vértice en el problema 1.

Figura prototípica de ángulos opuestos por el vértice: identificación de los segmentos RC y BT que intersecan en un punto P (ángulos opuestos por el vértice son iguales). De los 9 alumnos que en el problema 1 escogieron la subconfiguración $c, 7$ identificaron el punto de intersección de los segmentos TB y CR y sus ángulos correspondientes (ángulos opuestos por el vértice, Figura 5). La Figura 5 muestra la representación realizada por el estudiante en la que se muestra la identificación de ángulos opuestos por el vértice, obteniendo a continuación la subconfiguración $c$ (en esta respuesta se observa que el estudiante realiza además una rotación de la subconfiguración obtenida; falta representar $\triangle \mathrm{BPC}$ ).

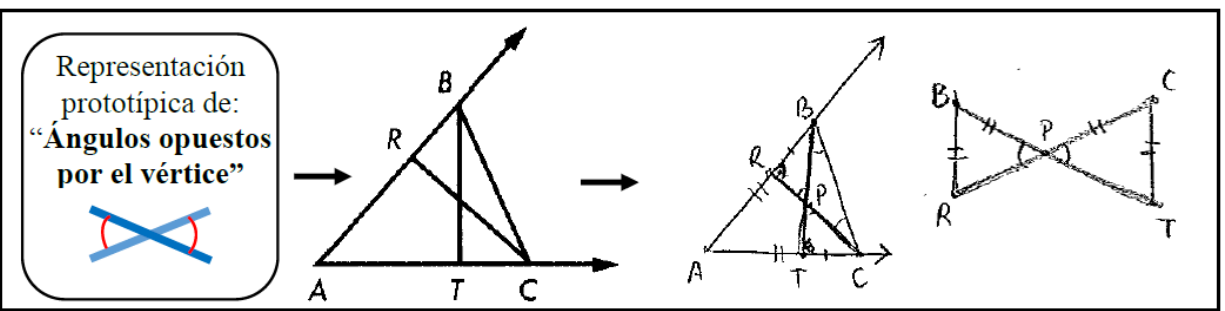

Figura 5 - Fragmento de la respuesta de un estudiante al problema 1 reflejando la influencia de la figura prototípica ángulos opuestos por el vértice.

Fuente: autores. 
- Caso 3: Influencia de la figura prototípica triángulo rectángulo en el problema 1. Figura prototípica de triángulo rectángulo: el resolutor percibe (erróneamente) en la figura inicial triángulos rectángulos, cree ver que $\mathrm{AC} \perp \mathrm{BT}$ y $\mathrm{RC} \perp \mathrm{AB}$.

En estos casos, el estudiante admite una propiedad exclusivamente por las características de la percepción visual de la figura inicial, priorizando el aspecto perceptual frente al formal. La Figura 6 muestra las representaciones realizadas por un estudiante y cómo identifica erróneamente triángulos rectángulos posiblemente por la influencia de la imagen prototípica triángulo rectángulo. Respectivamente: triángulo rectángulo-izquierda $(\triangle \mathrm{TAB}$; ángulo recto hacia la izquierda, obteniendo la subconfiguración $b$ ); y triángulo rectánguloderecha $(\triangle \mathrm{TBC}$; ángulo recto hacia la derecha, obteniendo la subconfiguración $a$, expresada mediante texto).

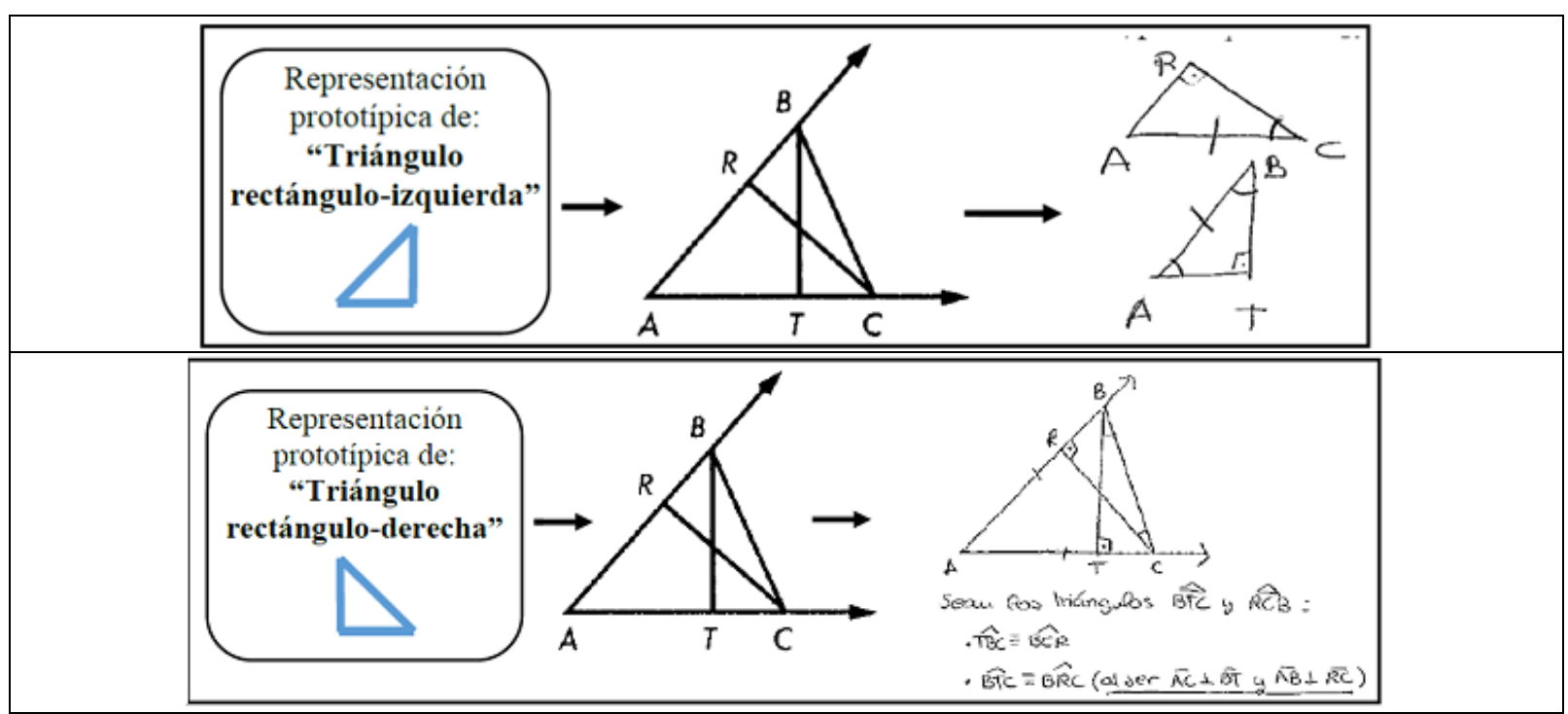

Figura 6 - Fragmento de las respuestas de dos estudiantes al problema 1 que refleja la influencia de la figura prototípica triángulo rectángulo.

Fuente: autores.

- Caso 4: Influencia de la figura prototípica triángulo rectángulo en el problema 2.

Figura prototípica de triángulo rectángulo: identifica (correctamente) en la figura inicial los triángulos rectángulos $\triangle \mathrm{ACM}$ y $\triangle \mathrm{AMN}$ definidos por los datos del problema ( $\triangle \mathrm{ACB}$ es un triángulo rectángulo en $\mathrm{C}$ y $\mathrm{MN} \perp \mathrm{AB}$ en $\mathrm{N}$ ); y de manera análoga al caso 1, realiza una rotación de una de las configuraciones $(\triangle \mathrm{AMN})$ representándola con los lados perpendiculares paralelos al borde del papel en que está dibujado.

La figura 7 muestra cómo un estudiante realiza la rotación del triángulo rectángulo $\triangle \mathrm{AMN}$ para representarlo en la posición prototípica (lados perpendiculares paralelos al borde del papel en que está dibujado), obteniendo la única subconfiguración relevante identificada. 


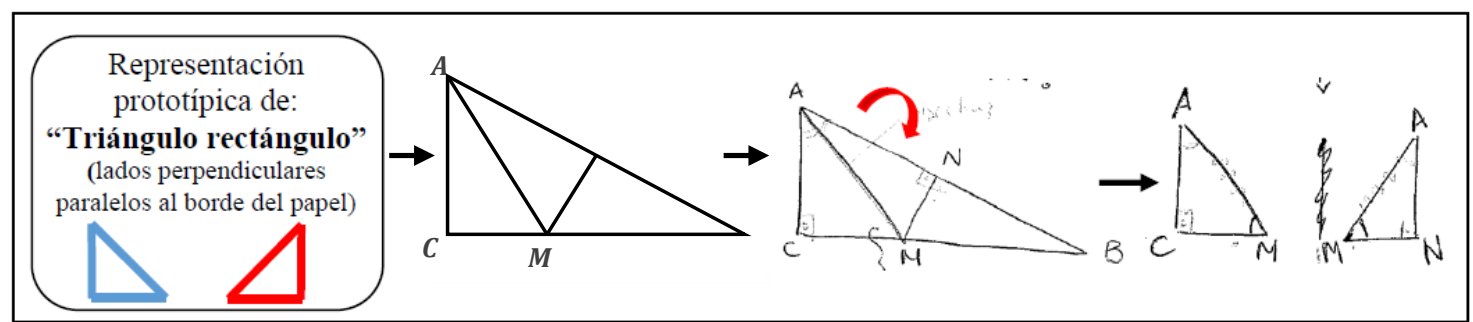

Figura 7 - Fragmento de la respuesta de un estudiante al problema 2 que refleja la influencia de la figura prototípica triángulo rectángulo.

Fuente: autores.

En el problema 1, 7 de los 9 estudiantes que escogieron la subconfiguración $c$, identificaron el punto de intersección de los segmentos TB y CR y sus ángulos correspondientes (representación prototípica de ángulos opuestos por el vértice). Además, 13 estudiantes admiten erróneamente en el problema 1 una propiedad $(\mathrm{AC} \perp \mathrm{BT}$ y $\mathrm{RC} \perp \mathrm{AB})$ para obtener la representación prototípica de triángulo rectángulo, iniciando a continuación la trayectoria de resolución vinculada a la subconfiguración a (10 estudiantes) y a la subconfiguración $b$ (3 estudiantes). Finalmente, en el problema 2, 18 alumnos realizan una rotación de una de las configuraciones (triángulo rectángulo $\triangle \mathrm{AMN}$ ), representándola con los lados perpendiculares paralelos al borde del papel para obtener la representación prototípica de triángulo rectángulo (Cuadro 3).

\section{Discusión y conclusiones}

Esta investigación estudia la relación entre la identificación de figuras prototípicas y el conocimiento de geometría en la resolución de problemas de probar como una manifestación del razonamiento configural. Los resultados muestran que la identificación de una configuración relevante activa determinados ítems de conocimiento geométrico, definiendo una determinada trayectoria de resolución, poniendo de manifiesto la importancia de las actividades de visualización en el aprendizaje de la geometría.

Nuestros resultados indican que la identificación de alguna subconfiguración relevante es un factor determinante en el desarrollo de una trayectoria de resolución, mostrando la relación entre la visualización y el conocimiento de geometría del resolutor. En esta situación podemos reconocer dos cambios de anclaje (DUVAL, 1998). Por una parte, podemos interpretar que las respuestas de los resolutores indican que el reconocimiento de una figura prototípica en la configuración inicial activa determinados conocimientos de geometría, poniendo de manifiesto el cambio del anclaje visual al anclaje discursivo descrito por Duval (1998). En segundo lugar, el conocimiento de geometría del resolutor parece que le permite 
visualizar unas configuraciones frente a otras, lo que pondría de manifiesto a su vez, el cambio del anclaje discursivo al anclaje visual. Las representaciones prototípicas (lo primero que ve el alumno) desempeñan un papel relevante en esta doble relación entre la percepción visual y el conocimiento vinculado a los cambios de anclaje visual-discursivo y viceversa (Figura 8).

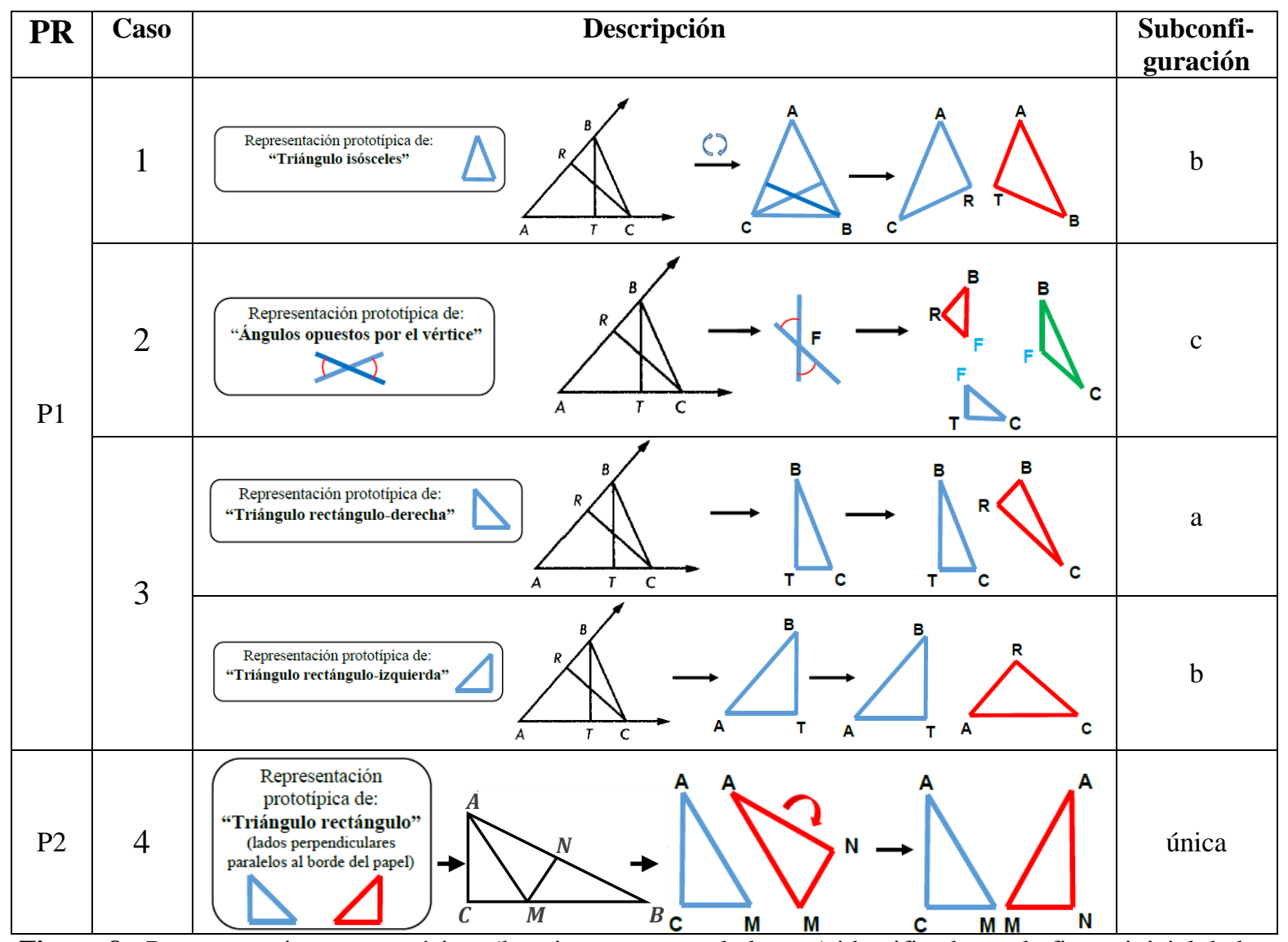

Figura 8 - Representaciones prototípicas (lo primero que ve el alumno) identificadas en la figura inicial de los problemas del cuestionario.

Fuente: autores.

Los datos relativos al número de estudiantes que en el problema 1 iniciaron una trayectoria de resolución y los conocimientos de geometría requeridos en cada una de ellas (Cuadro 2), parecen indicar la existencia de una relación entre la identificación de una determinada subconfiguración relevante de entre varias posibles y el conocimiento de geometría requerido para seguir la trayectoria vinculada a ella.

En el apartado de resultados solo hemos contabilizado los casos en los que existe evidencia en el discurso textual generado por el estudiante de la identificación de alguna figura prototípica. Por ejemplo, en el problema 1 marcando erróneamente como triángulo rectángulo una figura que no lo es. Sin embargo, podemos considerar esta idea de un modo más amplio, ya que el estudiante podría reconocer alguna de estas representaciones prototípicas, aunque no lo manifieste explícitamente. Es decir, si la representación prototípica 
de triángulo rectángulo para un determinado alumno es triángulo rectángulo-derecha (ángulo recto hacia la derecha), de los diversos triángulos rectángulos que podría percibir en la figura inicial (por ejemplo, $\triangle \mathrm{ABT}$ y $\triangle \mathrm{TBC}$ ) reconocerá, en primer lugar, el triángulo $\triangle \mathrm{TBC}$ ya que para él tiene mayor visibilidad, que le llevará a identificar preferentemente la subconfiguración $a$.

Los resultados de esta investigación indican la importancia de los procesos de visualización y de la relación entre las aprehensiones operativas y discursivas previos al desarrollo de los procesos deductivos formales. La identificación de una figura prototípica durante la resolución del problema permite centrar la atención del resolutor en sus propiedades figurales, es decir, como figuras percibidas por los sentidos, pero interpretadas a través del conocimiento de geometría previo. Así, la percepción visual se convierte en una herramienta anticipatoria en el proceso de resolución.

Estos resultados están en relación con los obtenidos por Gal y Linchevsky (2010) en el sentido de que la percepción visual vinculada a las aprehensiones operativas y discursivas pueden llegar a dificultar o favorecer el uso de determinados conceptos y propiedades geométricas necesarias para la resolución del problema. Desde estos resultados, se deriva la necesidad de enseñar a ver a los resolutores durante el aprendizaje de la geometría. Es decir, los maestros deberían enseñar a sus alumnos a ver las configuraciones geométricas que pueden ser relevantes para la resolución del problema.

Lo que nuestros resultados indican es que los estudiantes para maestro deben llegar a conocer la geometría en el ámbito curricular de la educación primaria, de forma que les permita ir más allá de simplemente reconocer propiedades y hechos geométricos en las figuras geométricas. De esta manera, el que el maestro sea consciente del papel que desempeña la visualización en el aprendizaje de la geometría le puede ayudar a gestionar las dificultades de sus estudiantes.

Lo anterior permite proponer una hipótesis de trabajo futuro. Si los maestros reconocen la importancia de la visualización en la resolución de problemas y conocen las relaciones entre diferentes hechos geométricos pueden llegar a gestionar, convenientemente, las dificultades de sus estudiantes cuando están aprendiendo conocimientos de geometría. Con este conocimiento especializado de geometría los maestros pueden proveer a sus alumnos de las destrezas necesarias para identificar subconfiguraciones en la figura inicial, que permitan activar los conocimientos de geometría oportunos para la resolución del problema (STYLIANIDES; BALL, 2008). Es decir, potenciar el papel de la visualización en la enseñanza - aprendizaje de la geometría se convierte en un aspecto clave. Sin embargo, es 
necesaria más investigación para apoyar esta línea de trabajo centrada en la relación entre la visualización, la generación del razonamiento configural y la resolución de problemas de probar en el sentido de aportar información específica para la toma de decisiones en la formación de maestros.

\section{Reconocimientos}

Esta investigación ha recibido el apoyo de los Proyectos I+D+i, EDU2011-27288 y EDU2014-54526-R del Ministerio de Ciencia e Innovación, España.

Una versión previa de este trabajo fue presentada en CIAEM-2015, Chiapas-México, 3-7 de mayo 2015.

\section{Referencias}

BATTISTA, M. T. The development of geometric and spatial thinking. In: LESTER, F. K. (Ed.). Second Handbook of Research on Mathematics Teaching and Learning. Charlotte: Ed. Information Age Publishing. NCTM, 2007. p. 843-908.

CHEN, C. L.; HERBST, P. The interplay among gestures, discourse, and diagrams in students' geometrical reasoning. Educational Studies in Mathematics, Holanda, v. 83, n. 2, p. 285-307, jun. 2013.

CHINNAPPAN, M. Schemas and mental models in geometry problem solving. Educational Studies in Mathematics, Holanda, v. 36, n. 3, p. 201-217, sep. 1998.

CHINNAPPAN, M.; LAWSON, M. A framework for analysis of teachers' geometric content knowledge and geometric knowledge for teaching. Journal of Mathematics Teacher Education, Holanda, v. 8, n. 3, p. 197-221, jun. 2005.

CLEMENTE, F.; LLINARES, S. Conocimiento de geometría especializado para la enseñanza en Educación Primaria. In: BERCIANO, A. et al. (Ed.). Investigación en Educación Matemática XVII. Bilbao: Ed. SEIEM, 2013. p. 229-236.

CLEMENTE, F.; LLINARES, S. Relación entre el conocimiento de geometría y el "truncamiento" del razonamiento configural. In: GONZÁLEZ, M. T. et al. (Ed.). Investigación en Educación

Matemática XVIII. Salamanca: Ed. SEIEM, 2014. p. 247-256.

CLEMENTE, F.; LLINARES, S. Formas del discurso y razonamiento configural de estudiantes para maestro en la resolución de problemas de geometría. Enseñanza de las Ciencias, Barcelona, v. 33, n. 1, p. 9-27, 2015.

CLEMENTE, F.; TORREGROSA, G.; LLINARES, S. La identificación de figuras prototípicas en el desarrollo del razonamiento proporcional. In: SCOTT, P.; RUIZ, A. (Org.). Educación Matemática en las Américas. México: CIAEM, 2015. p. 130-140. Disponible en: $<$ http://ciaemredumate.org/memorias-ciaem/xiv/pdf/Vol9Geom.pdf>. Acceso en: 24 oct. 2016. 
DUVAL, R. Geometrical pictures: kinds of representation and specific processes. In: SUTHERLAND, R.; MASON, J. (Ed.). Exploiting Mental Imagery with Computers in Mathematical Education. Berlin: Ed. Springer, 1995. p. 142-157.

DUVAL, R. Geometry from a cognitive point of view. In: MAMMANA, C.; VILLANI, V. (Ed.). Perspectives on the Teaching of Geometry for the 21st Century: an ICMI Study. Dordrecht: Ed. Kluwer, 1998. p. 37-52.

DUVAL, R. Representation, vision and visualization: cognitive functions in mathematical thinking. Basis issues for learning. In F. Hitt \& M. Santos (Eds.), ANNUAL MEETING NORTH AMERICAN CHAPTER OF THE INTERNATIONAL GROUP OF PME, 21., 1999, Cuernavaca, México. Proceedings of the annual Meeting PME-NA. Columbus, Ohio, USA: ERIC/CSMEE Publications, 1999. p. 3-26.

DUVAL, R. Cognitive functioning and the understanding of mathematical processes of proof. In: BOERO, P. (Ed.). Theorems in School. From History, Epistemology and Cognition to Classroom Practice. Rotterdam: Ed. Sense Publishers, 2007. p. 137-162.

FISCHBEIN, E. The theory of figural concepts. Educational Studies in Mathematics, Holanda, v. 24, n. 2, p. 139-162, 1993.

GAL, H.; LINCHEVSKI, L. To see or not to see: analyzing difficulties in geometry from the perspective of visual perception. Educational Studies in Mathematics, Holanda, v.74, n. 2, p. 163 $183,2010$.

HANNA, G.; SIDOLI, N. Visualisation and proof: a brief survey of philosophical perspectives. ZDM Mathematics Education, Dordrecht, v.39, n. 1-2, p. 73-78, 2007.

HERBST, P. Interactions with Diagrams and the Making of Reasoned Conjectures in Geometry. ZDM Mathematics Education, Dordrecht, v. 36, n. 5, p. 129-139, 2004.

HERBST, P. et al. "Doing Proofs" in geometry classrooms. In: STYLIANOU, D.; BLANTON, M.L.; E. KNUTH, J. (Ed.). Teaching and Learning Proof Across the Grades: A K-16 Perspectives. New York: Ed. Routledge, 2009. p. 250-268.

HERSHKOWITZ, R. Visualization in geometry: two sides of the coin. Focus on Learning Problems in Mathematics, USA, v. 11, n. 1, p. 61-76, 1989.

HERSHKOWITZ, R. Psychological aspects of learning geometry. In: NESHER, P.; KILPATRICK, J. (Ed.). Mathematics and Cognition. Cambridge: Ed. Cambridge University Press, 1990. p. 70-95.

HERSHKOWITZ, R.; PARZYSZ, B.; VAN DORMOLEN, J. Space and shape. In: BISHOP, A. et al. (Ed.). International Handbook of Mathematics Education: part 1. Dordrecht: Ed. Kluwer, 1996. p. 161-204.

HOLLEBRANDS, K.; LABORDE, C.; STRÁBER, R. Technology and the learning of geometry at the secondary level. In: HEID, M.K.; BLUME, G.W. (Ed.). Research on Technology and the Teaching and Learning of Mathematics: vol. 1. USA: Ed. NCTM, 2008. p. 155-205.

LLINARES, S.; CLEMENTE, F. Characteristics of pre-service primary school teachers' configural reasoning. Mathematical Thinking and Learning, Oxford, v. 16, n. 3, p. 234-250, 2014.

MESQUITA, A. L. On conceptual obstacles linked with external representation in geometry. Journal of Mathematical Behavior, Amsterdam, v. 17, n. 2, p. 183-195, 1998. 
MOISE, E.; DOWNS, F. Geometría Moderna. Delaware, E.U.A.: Addison-Wesley Iberoamericana, 1986.

PRESMEG, N. Research on visualization in learning and teaching mathematics. In: GUTIÉRREZ, A.; BOERO, P. (Ed.). Handbook of Research on the Psychology of Mathematics Education: past, present and future. Rotterdam/Taipei: Ed. Sense Publishers, 2006. p. 205-236.

PRIOR, J.; TORREGROSA, G. Razonamiento configural y procedimientos de verificación en contexto geométrico. Revista Latinoamericana de Investigación en Matemática Educativa, México, v. 16, n. 3, p. 339-368, 2013.

PRUSAK, N.; HERSHKOWITZ, R.; SCHWARZ, B. B. From visual reasoning to logical necessity through argumentative design. Educational Studies in Mathematics, Holanda, v.79, n. 1, p.19-40, 2012.

STYLIANIDES, G. J.; BALL, D. L. Understanding and describing mathematical knowledge for teaching: knowledge about proof for engaging students in the activity of proving. Journal of Mathematics Teacher Education, Dordrecht, v. 11, p. 307-332, 2008.

TORREGROSA, G.; QUESADA, H. Coordinación de procesos cognitivos en Geometría. Revista Latinoamericana de Investigación en Matemática Educativa, México, v. 10, n. 2, p. 275-300, 2007.

TORREGROSA, G.; QUESADA, H.; PENALVA, M. C. Razonamiento configural como coordinación de procesos de visualización. Enseñanza de las Ciencias, Barcelona, v. 28, n. 3, p. 327 340, 2010.

VINNER, S.; HERSHKOWITZ, R. Concept images and common cognitive paths in the development of some simple geometrical concepts. In: KARPLUS, R. (Ed.). CONFERENCE OF THE

INSTERNATIONAL GROUP FOR THE PSYCHOLOGY OF MATHEMATICS EDUCATION, 4., 1980, Berkley. Proceedings of the 4th PME conference. Berkeley: Lawrence Hall of Science, 1980. p. $177-184$.

Submetido em Junho de 2016. Aprovado em Novembro de 2016. 